\title{
SELDI-TOF-MS in chronic obstructive pulmonary disease
}

\author{
ZHANG Xia ${ }^{1}$, ZHANG Jie $^{2 *}$, LI Qi $^{1^{*}}$, WU XiaoYi ${ }^{3}$, WANG Ting $^{2} \&$ WANG YuLing ${ }^{2}$ \\ ${ }^{1}$ Beijing Chest Hospital, Capital Medical University; Beijing Tuberculosis and Thoracic Tumor Research Institute, Beijing 101149, China; \\ ${ }^{2}$ Department of Respiratory Diseases, Beijing Tiantan Hospital, Capital Medical University, Beijing 100050, China; \\ ${ }^{3}$ Beijing De Yi Biosystems Inc, Beijing 100035, China
}

Received February 15, 2012; accepted July 11, 2012; published online September 16, 2012

\begin{abstract}
Chronic obstructive pulmonary disease (COPD) has a profound impact on daily life, yet remains underdiagnosed and undertreated. This study aims to discover potential protein biomarkers for diagnosis and classification of COPD. Fifty-seven COPD patients and 40 controls were divided into a training set (30 COPD patients, 20 healthy controls) and a test set (27 COPD patients, 20 healthy controls). Serum proteomic profiles were measured using surface-enhanced laser desorption/ionization time-of-flight mass spectrometry (SELDI-TOF-MS). A classification tree was established using Biomarker Pattern Software (BPS). Next we screened distinct proteins present in patients with acute exacerbations of COPD (AECOPD), stable COPD and healthy controls, in order to establish diagnostic models for classification of COPD. Twenty peaks showed statistically significant differences between COPD patients and healthy controls $(P<0.05)$. Two proteomic peaks $(3167$ and $5477 \mathrm{~m} / \mathrm{z}$ ) were chosen by BPS to establish a classification tree in the training set. The sensitivity and specificity of this classification tree were $92.59 \%$ and $90.00 \%$ respectively in the testing set. Furthermore, differently expressed proteins were detected among the patients with AECOPD, stable COPD, and healthy controls. Two protein profiles ( 3167 and $4645 \mathrm{~m} / \mathrm{z}$ ) could distinguish between stable COPD patients and healthy controls. Three protein profiles (3167, 2963 and $2973 \mathrm{~m} / \mathrm{z}$ ) could distinguish between AECOPD patients and healthy controls. Three protein profiles $(5476,14039$ and $2831 \mathrm{~m} / \mathrm{z}$ ) could distinguish between stable COPD patients and AECOPD patients. SELDI-TOFMS Proteinchip technology is a quick, easy and practical, high throughput analytic method. It shows the diagnostic models established by distinguished proteomic peaks can discriminate COPD patients from healthy control and can identify different stages of COPD. It will provide a highly accurate approach for diagnosis and clinical staging of COPD.
\end{abstract}

chronic obstructive pulmonary disease, surface-enhanced laser desorption/ionization time-of-flight mass spectrometry, proteomics

Citation: $\quad$ Zhang X, Zhang J, Li Q, et al. SELDI-TOF-MS in chronic obstructive pulmonary disease. Chin Sci Bull, 2013, 58: 634-640, doi: 10.1007/s11434-012-5460-0

Chronic Obstructive Pulmonary Disease (COPD) is recognized as a major public health problem with increasing morbidity and mortality [1]. It has been forecasted that COPD will be ranked third as a cause of mortality by 2020 , and the prevalence of COPD is most often reported in the range of $6 \%-10 \%$ of the total adult population [2,3]. COPD is characterised by airflow limitation that is progressive and not fully reversible [4]. The onset of COPD is generally insidious, with slowly evolving symptoms, and the perception of these symptoms as 'self-inflicted' by patients and physicians may lead to significant delay in medical consul-

*Corresponding authors (email: zhang2001512@126.com; yixiao6561@126.com) tation and initiation of therapy [5,6]. Early diagnosis of this disease is a challenge for the coming years [7]. Repeated acute exacerbation COPD (AECOPD) is a major public health problem. They are important clinical events and have prognostic signifcance [8], so study of AECOPD is one of the practical problems we need to face. At present, diagnostic criteria for AECOPD has not yet been standardized, and there is no uniform diagnostic criteria to distinguish between stable COPD and AECOPD in China. In the traditional way, classification is based on clinical features. To date, no specific biological markers have been used to identify this disease.

Recent advances in proteomic analysis have offered ex- 
citing opportunities for finding novel biomarkers in biological fluids, especially in sera [9-13]. Surface-assisted laser desorption/ionization time-of-flight mass spectrometry (SELDI-TOF-MS) offers a rapid and robust approach for protein biomarker discovery without the need for purification and separation of proteins prior to analysis $[14,15]$. It has proved to be a powerful tool for potential biomarker discovery [16-22]. Findings from previous studies suggest that SELDI-TOF-MS may facilitate detection of COPDassociated serum biomarkers [23]. However, larger cohorts will be needed to validate these biomarkers and determine their predictive value in longitudinal studies. And to date, there are no published reports evaluating the efficacy of SELDI-TOF-MS for distinguishing stable COPD patients from AECOPD patients.

In this study, we first used SELDI-TOF-MS technology to screen potential protein patterns specific to COPD, and next, to screen distinct proteins in different stages of COPD in order to establish diagnostic models for clinical staging of COPD.

\section{Materials and methods}

\subsection{Sample collection}

Serum samples were collected from the Department of Respiratory Diseases at Beijing Tiantan Hospital, Capital Medical University, China. Patients with COPD were diagnosed according to the Global Initiative for Obstructive Lung Disease (GOLD) criteria. This study included 97 specimens, 57 samples of which were obtained from COPD patients and 40 samples which were obtained from healthy controls in the medical center of Beijing Tiantan Hospital during routine examinations (Table 1). COPD patients (baseline $\mathrm{FEV}_{1},(49.6 \pm 17.2) \%$ of predicted) fulfilled GOLD criteria with $\mathrm{FEV}_{1} / \mathrm{FVC}<70 \%$. Healthy controls were without pulmonary disease and $\mathrm{FEV}_{1} / \mathrm{FVC}>80 \%$. COPD patients were separated into stable COPD patients and AECOPD patients, according to GOLD criteria. We obtained informed consent from all subjects and approval from the Beijing Tiantan Hospital ethical committee.

The specimens were separated into two groups: (1) the training group, with 30 COPD patients (15 stable COPD patients, 15 AECOPD patients) and 20 healthy controls, was used to establish the diagnosis model (Model I) to distinguish between COPD patients and healthy controls. (2) The testing group, with 27 COPD patients (15 stable COPD patients, 12 AECOPD patients) and 20 healthy controls, was analyzed to validate the accuracy and validity of the diagnosis model (Model I) derived from the training group.

Thirty cases of stable COPD patients and 30 healthy controls (30 cases of healthy controls were selected from the 40 cases of healthy controls, and age and sex-matched to the stable COPD patients) were used to establish the diagnosis model (Model II) to distinguish between stable COPD
Table 1 Characteristics of COPD patients and healthy controls

\begin{tabular}{ll}
\hline Characteristics & Number \\
\hline COPD patients & $66(51-85)$ \\
Mean age (age range) & 32 \\
Sex & 25 \\
Male & \\
Female & 30 \\
Stage & 27 \\
$\quad$ Stable COPD patients & \\
AECOPD patients & $62(51-76)$ \\
Stable COPD patients & \\
$\quad$ Mean age (age range) & 24 \\
$\quad$ Sex & 6 \\
Male $\quad$ & $70(56-85)$ \\
Female & \\
AECOPD patients & 19 \\
$\quad$ Mean age (age range) & \\
Sex & $64(50-80)$ \\
Male & 24 \\
Female & 16 \\
Mealthy controls age (age range) & \\
Sex & \\
Male &
\end{tabular}

patients and healthy controls. Twenty-seven cases of AECOPD patients and 27 healthy controls (27 cases of healthy controls were selected from the 40 cases of healthy controls, and age and sex-matched with AECOPD patients) were used to establish the diagnosis model (Model III) to distinguish between stable AECOPD patients and healthy controls; 15 cases of stable COPD patients and 15 cases of AECOPD patients (15 cases of stable COPD patients were selected from the 30 cases of stable COPD patients, and 15 cases of AECOPD patients were selected from the 30 cases of AECOPD patients and age and sex-matched with stable COPD patients) were used to establish the diagnosis model (Model IV) to distinguish between stable COPD patients and AECOPD patients.

All blood samples were collected in the morning before breakfast. They were stored at $4^{\circ} \mathrm{C}$ for $2 \mathrm{~h}$ and then centrifuged at $3500 \mathrm{r} / \mathrm{min}$ for $5 \mathrm{~min}$. All serum samples were stored at $-80^{\circ} \mathrm{C}$ for further analysis.

\subsection{SELDI protein profiling}

Serum samples were thawed on ice and centrifuged at $10000 \mathrm{r} / \mathrm{min}$ for $2 \mathrm{~min}$. A total of $20 \mu \mathrm{L}$ of U9 buffer ( $9 \mathrm{~mol} / \mathrm{L}$ urea, $2 \%$ CHAPS, $2 \mathrm{mmol} / \mathrm{L}$ DTT and $50 \mathrm{mmol} / \mathrm{L}$ Tris-HCl, pH 9) (Sigma, Santa Clara, USA) were added to $10 \mu \mathrm{L}$ of each serum sample, and the solutions were vortexed for $30 \mathrm{~min}$. The resulting serum solutions were further diluted by $360 \mu \mathrm{L}$ of buffer solution $(50 \mathrm{mmol} / \mathrm{L}$ sodium acetate, $\mathrm{pH}$ 4.0) (Sigma). Weak Cation Exchange protein chips, CM10 (Ciphergen Biosystems, Fremont, USA) were pre-equilibrated with $200 \mu \mathrm{L}$ of buffer solution twice. Next, $100 \mu \mathrm{L}$ of diluted serum sample was added to each spot of the CM10 protein chip in a bioprocessor (Ciphergen Bio- 
systems). The bioprocessor was then sealed and agitated on a platform shaker for $60 \mathrm{~min}$ at $4^{\circ} \mathrm{C}$. After discarding the remaining samples, the chips were washed with $200 \mu \mathrm{L}$ of buffer solution for $5 \mathrm{~min}$. This procedure was repeated twice, with a brief water rinse after application of buffer solution. The chips were then removed from the bioprocessor and air-dried. Each spot on the chip received two applications of $0.5 \mu \mathrm{L}$ of a saturated sinapinic acid in $50 \%(\mathrm{v} / \mathrm{v})$ acetontrile and $0.5 \%(\mathrm{v} / \mathrm{v})$ trifluoroacetic acid (Sigma), and the chips were air-dried. ProteinChip Arrays were read by surface-enhanced laser desorption/ionization time-of-flight mass spectrometry (Ciphergen Biosystems). The optimal detection parameter of mass/charge size range was set between 2000 and $20000 \mathrm{~m} / \mathrm{z}$ with a maximum of $50000 \mathrm{~m} / \mathrm{z}$. The laser intensity was set at 150 and detector sensitivity was set at 8 . The all-in-one peptide molecular mass standard (Ciphergen Biosystems) was used to generate a peptide standard spectrum for mass accuracy calibration.

\subsection{Bioinformatics and biostatistics}

Details of classification and regression tree analysis have been previously described [24]. The SELDI-TOF-MS were obtained and analyzed using Ciphergen Protein Chip Software 3.1.1. Peak labeling was performed by the Biomarker Wizard Software. The $P$ value for statistical significance was set at 0.05 . The intensities of selected peaks were subsequently transferred to BPS to construct the classification tree of all.

\section{Results}

\subsection{Comparison of serum protein spectra between COPD patients and healthy controls in the training set}

Serum samples from the training set were evaluated by comparing the results obtained by SELDI-TOF-MS with those from the CM10 chip. Twenty peaks showed statistically significant differences between COPD patients and healthy controls $(P<0.05)$ (Table 2$)$. Among them, 8 protein peaks $(3270,3514,9315,9369,9430,11501,11545$ and $11717 \mathrm{~m} / \mathrm{z}$ ) were up-regulated and 12 protein peaks (2086, 2880, 2944, 2973, 3167, 3215, 3937, 4093, 4176, 5439,5477 and $5921 \mathrm{~m} / \mathrm{z}$ ) were down-regulated in serum samples from patients with COPD. Figure 1 shows one protein peak $(3167 \mathrm{~m} / \mathrm{z})$ was down-regulated in COPD patients.

\subsection{Decision tree classification (Model I)}

To set up a classification tree as a diagnostic model, the intensities of protein peaks in the training set were transferred to BPS. A total of two peaks (3167 and $5477 \mathrm{~m} / \mathrm{z}$ ) were automatically selected to construct a classification tree (Figure 2). All 50 cases in the training set entered the classification tree from the root node in the first layer, which
Table 2 The average peak intensity of 19 distinct protein spectra found in sera of COPD patients and healthy controls $(P<0.05)$

\begin{tabular}{rccr}
\hline$m / z$ & $P$ & COPD & Controls \\
\hline 3167 & $4.60 \times 10^{-9}$ & $3.58 \pm 1.9$ & $11.13 \pm 4.37$ \\
5477 & $1.00 \times 10^{-6}$ & $3.77 \pm 3.15$ & $9.64 \pm 2.47$ \\
3937 & $1.82 \times 10^{-6}$ & $11.12 \pm 5.13$ & $23.40 \pm 8.50$ \\
4176 & $1.58 \times 10^{-6}$ & $3.37 \pm 1.30$ & $6.91 \pm 3.24$ \\
11717 & $2.30 \times 10^{-4}$ & $1.96 \pm 2.28$ & $0.55 \pm 0.23$ \\
11545 & $9.42 \times 10^{-4}$ & $1.02 \pm 1.10$ & $0.35 \pm 0.16$ \\
2944 & $1.16 \times 10^{-3}$ & $4.56 \pm 1.70$ & $7.98 \pm 4.86$ \\
2880 & $2.97 \times 10^{-3}$ & $5.75 \pm 3.89$ & $8.89 \pm 3.74$ \\
11501 & $8.44 \times 10^{-3}$ & $1.03 \pm 1.35$ & $0.34 \pm 0.14$ \\
3514 & 0.015 & $2.64 \pm 2.87$ & $0.83 \pm 1.40$ \\
9369 & 0.018 & $5.22 \pm 4.15$ & $2.46 \pm 1.20$ \\
4093 & 0.029 & $51.32 \pm 10.54$ & $59.56 \pm 13.31$ \\
3215 & 0.030 & $2.32 \pm 2.03$ & $4.32 \pm 4.02$ \\
2973 & 0.034 & $3.91 \pm 2.53$ & $5.43 \pm 2.58$ \\
9315 & 0.037 & $6.57 \pm 2.99$ & $4.74 \pm 1.64$ \\
3270 & 0.039 & $6.27 \pm 3.28$ & $5.05 \pm 4.58$ \\
5921 & 0.043 & $20.37 \pm 9.18$ & $25.54 \pm 9.30$ \\
5349 & 0.045 & $2.30 \pm 2.26$ & $4.22 \pm 3.65$ \\
2086 & 0.049 & $6.75 \pm 3.48$ & $8.50 \pm 3.12$ \\
9430 & 0.050 & $5.94 \pm 4.13$ & $3.45 \pm 1.85$ \\
\hline
\end{tabular}

splited into two nodes in the second layer, according to the peak intensity with a $\mathrm{m} / \mathrm{z}$ value of $3167 \mathrm{~m} / \mathrm{z}$. Twenty-eight cases were assigned to terminal node 1 , because their peak intensity value was $\leqslant 5.982$, while 22 cases were assigned to node 2 , because their peak intensity value was $>5.982$. In the third layer, the 22 cases in node 2 were classified into terminal node 2 (COPD patients) and terminal node 3 (healthy controls) according to the peak intensity with a $\mathrm{m} / \mathrm{z}$ value of $5477 \mathrm{~m} / \mathrm{z}$. The classification tree using the combination of these two protein peaks identified 30 COPD patients and 20 healthy controls, resulting in a sensitivity of $100 \%(30 / 30)$, a specificity of $100 \%(20 / 20)$ and an accuracy rate of $100 \%(50 / 50)$ respectively (Table 3$)$.

\subsection{Decision tree classification (Model I) validation}

To validate the accuracy of the classification model derived from the training set, we applied the derived classification tree to a test data set consisting of samples from 27 COPD patients and 20 healthy controls. The classification tree discriminated the COPD samples from the controls with a sensitivity of $92.59 \%(25 / 27)$, a specificity of $90.00 \%(18 / 20)$, and a accuracy rate of $91.49 \%$ (43/47) (Tables 3$)$. The area under the receiver operating characteristics (ROC) curve of this model was 0.913 (Figure 3).

\subsection{Decision tree classification (Model II)}

Nineteen peaks showed statistically significant differences between the 30 stable COPD patients and 30 healthy controls 


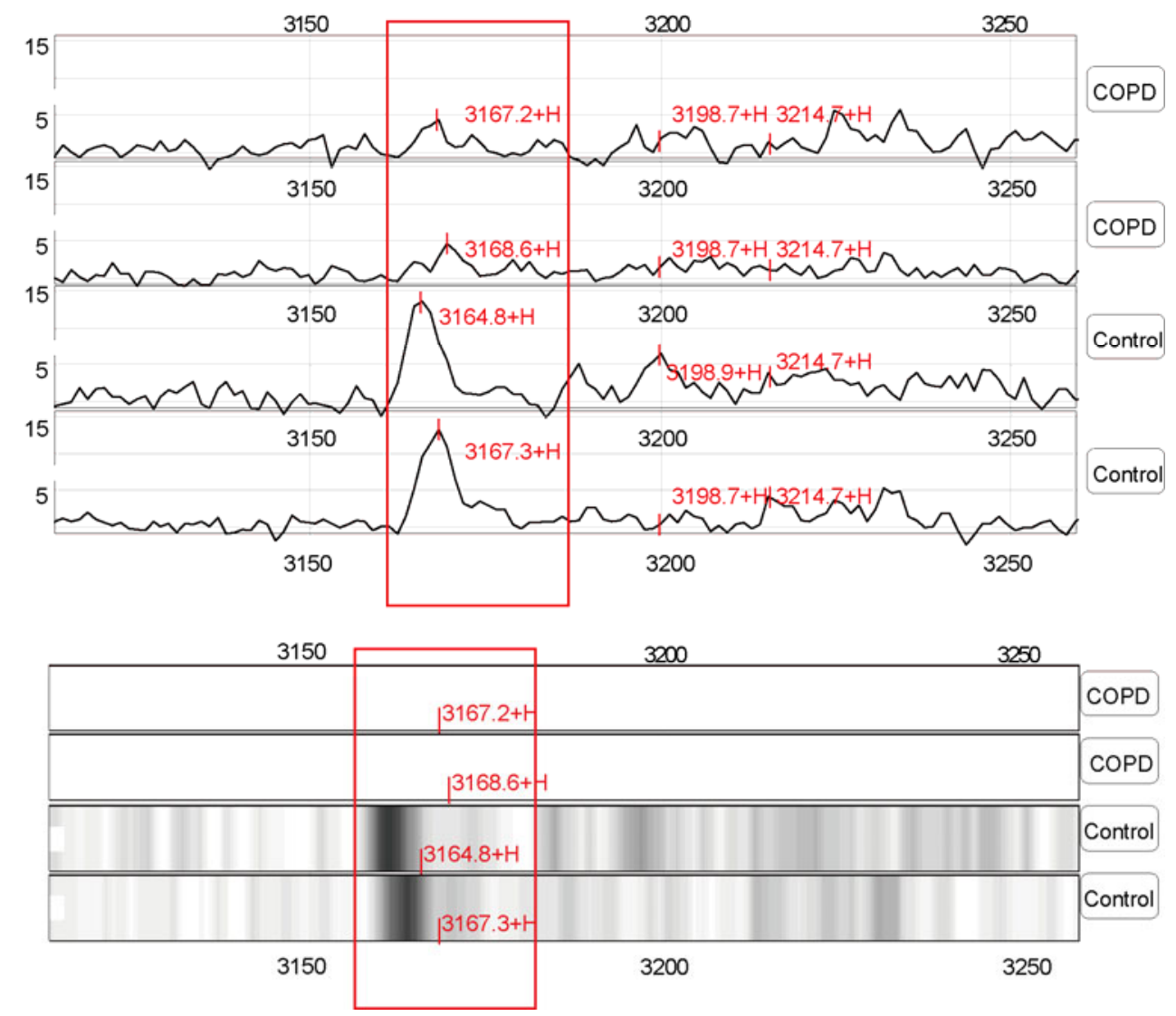

Figure 1 Detection of protein of mass pattern in sera. Mass spectra of serum samples from two COPD patients and two health controls. One protein peak (3167 $\mathrm{m} / \mathrm{z}$ ) was down-regulated in COPD patients. $3167 \mathrm{~m} / \mathrm{z}$ was a median calculated by Biomarker Wizard Software, and the floating range was less than $0.3 \%$.

Table 3 Performance of of the classification tree analysis ( Model I ) in the training and testing sets

\begin{tabular}{cccc}
\hline Sets & Groups & Sensitivity $(\%)$ & Specificity $(\%)$ \\
\hline Training set & 30 COPD/ 20 controls & 100 & 100 \\
Testing set & 27 COPD/ 20 controls & 92.59 & 100 \\
91.47 & 90.00 \\
\hline
\end{tabular}

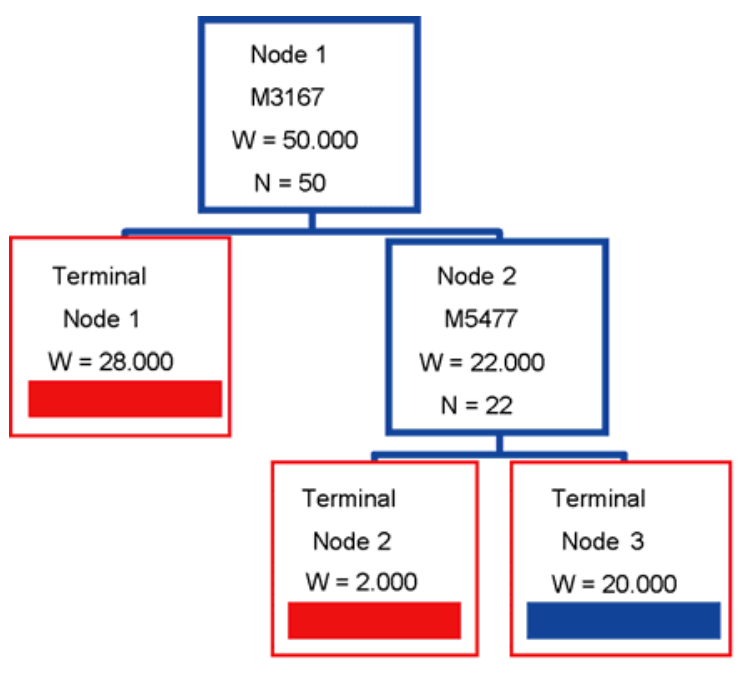

Figure 2 Classification of COPD patients (red) and healthy controls (blue) in the training set.

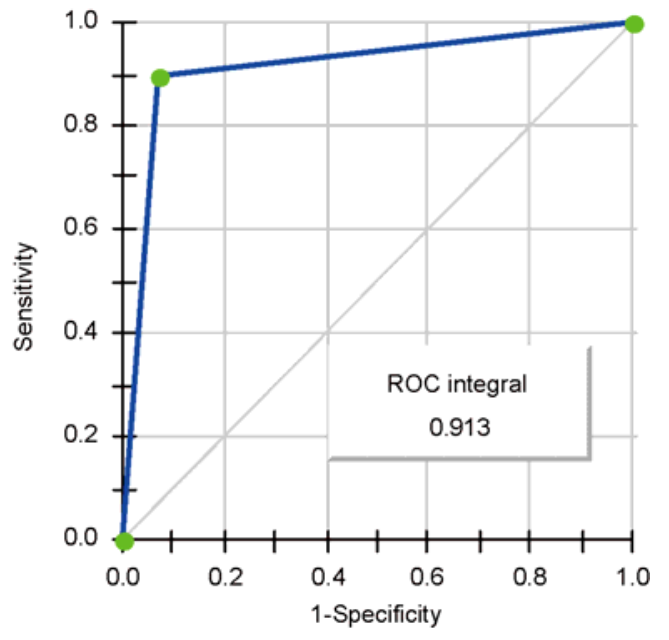

Figure 3 The area under ROC curve of Model I. 
$(P<0.05)$. Among them, four protein peaks $(2069,9367$, 9434 and $11510 \mathrm{~m} / \mathrm{z}$ ) were up-regulated and 15 protein peaks $(2702,2880,2944,3167,3936,4176,4297,4474$, $4645,5299,5380,5477,7777,7986$ and $14775 \mathrm{~m} / \mathrm{z}$ ) were down-regulated in serum samples from patients with stable COPD. Two peaks (3167 and $4645 \mathrm{~m} / \mathrm{z}$ ) were automatically selected to construct a classification tree that could significantly distinguish between stable COPD patients and controls. The classification tree using the combination of these two protein peaks identified 30 stable COPD patients and 30 healthy controls, resulting in a sensitivity of $96.67 \%$ (29/30) and a specificity of 96.67\% (29/30) (Table 4).

\subsection{Decision tree classification (Model III)}

Twenty-four peaks showed statistically significant differences between 27 AECOPD patients and 27 healthy controls $(P<0.05)$. Among them, 13 protein peaks $(2963,3271$, 3514, 5802, 5836, 6115, 9312, 9371, 9434, 9731, 11445, 11519 and $11730 \mathrm{~m} / \mathrm{z}$ ) were up-regulated and 11 protein peaks $(2558,2880,2944,2973,3167,3937,4283,4297$, 4649, 5477 and $14049 \mathrm{~m} / \mathrm{z}$ ) were down-regulated in serum samples of patients with AECOPD. Three peaks (3167, 2963 and $2973 \mathrm{~m} / \mathrm{z}$ ) were automatically selected to construct a classification tree that could significantly distinguish between AECOPD and controls. The classification tree using the combination of these three protein peaks identified 27 stable COPD patients and 27 healthy controls, resulting in a sensitivity of $100 \%(27 / 27)$ and a specificity of $100 \%$ (27/27) (Table 4).

\subsection{Decision tree classification (Model IV)}

Eight peaks showed statistically significant differences between 15 stable COPD patients and 15 AECOPD patients $(P<0.05)$. They $(2831,3936,5335,5476,7776,7982,9312$ and $14039 \mathrm{~m} / \mathrm{z}$ ) were all up-regulated in serum samples of patients with AECOPD. Three peaks (5476, 14039 and $2831 \mathrm{~m} / \mathrm{z}$ ) automatically selected to construct a classification tree could significantly distinguish between stable COPD and AECOPD. The classification tree using the combination of these three protein peaks identified 15 stable COPD patients and 15 AECOPD patients, resulting in a sensitivity of $93.33 \%$ and a specificity of $100 \%$ respectively (Table 4). One in 15 stable COPD samples was misclassified.

\subsection{Comparison of serum protein spectra between sta- ble COPD, AECOPD patients and healthy controls}

Nineteen peaks showed statistically significant differences between stable COPD, AECOPD patients and healthy controls $(P<0.05)$. Among them, intensities of proteins with 5802, 5839, 9371, 9731 and $11445 \mathrm{~m} / \mathrm{z}$ were orderly increasing from healthy controls, stable COPD to AECOPD. Intensity of protein with $\mathrm{m} / \mathrm{z} 2303$ was orderly reducing from healthy controls, stable COPD to AECOPD. Intensities of proteins with $2880,3167,3936,4140,4297,4645$, 5478,5921 and $6451 \mathrm{~m} / \mathrm{z}$ were orderly reducing from healthy controls, AECOPD to stable COPD.

\section{Discussion}

In this study, we obtained serum protein mass spectra from COPD patients and healthy controls using SELDI-TOF-MS. Twenty peaks had statistically significant differences between 30 COPD patients and 20 healthy controls (the training set. Among them, eight protein peaks were up-regulated and 12 were down-regulated in serum samples of patients with COPD). Up-regulated proteins may be those which promote the occurrence and development of COPD; down-regulated proteins may be those conducive to the maintenance of lung structure and function. Identification of these proteins will help understand the pathogenesis of COPD. We used a two-step approach for proteomic biomarker screening. To translate from proteomic peaks to proteomic signatures for discriminating COPD patients from healthy controls, we utilized BPS to select the best proteomic peaks for construction of classification tree [25]. The classification tree using the combination of the two peaks $(3167$ and $5477 \mathrm{~m} / \mathrm{z}$ ) identified 30 COPD and 20 healthy subjects with a calculated sensitivity of $100 \%$ and a specificity of $100 \%$. To validate the accuracy and validity of the classification model derived from the training set, we applied the derived classification tree to a test dataset consisting of 27 COPD and 20 control samples. The classification tree discriminated the COPD samples from the controls with a sensitivity of $92.59 \%$ and a specificity of $90.00 \%$. So we consider that SELDI-TOF-MS technology can detect several specific markers from COPD sera that have clinical significance in the diagnosis of COPD. The two proteins selected using this technique $(3167,5477 \mathrm{~m} / \mathrm{z}$ ) may play an important role in the pathogenesis of COPD.

Table 4 Performance of the classification tree analysis (models II-IV)

\begin{tabular}{llcc}
\hline Models & \multicolumn{1}{c}{ Groups } & Sensitivity (\%) & Specificity (\%) \\
\hline Model II & 30 Stable COPD/30 controls & 96.67 & 96.67 \\
Model III & 27 AECOPD/27 controls & 100 & 100 \\
Model IV & 15 COPD/15 AECOPD & 93.33 & 100 \\
\hline
\end{tabular}


Currently, in literature, few SELDI-TOF-MS studies on COPD are reported, especially performed on serum samples. Bowler et al. [23] identified a panel of five biomarkers using the IMAC protein chip that could distinguish COPD patients from controls with sensitivity and specificity of $91.67 \%$ and $88.33 \%$. Our and Bowler's studies have demonstrated the feasibility of using SELDI as a diagnostic test for COPD, and that the serum protein fingerprint of COPD patients is different from that of healthy people. This permits to reveal low abundance proteins in serum and plasma samples, as needed for biomarker discovery [26-28]. However, our research results differ from those of Bowler's study. Different experimental conditions, a different chip choice, different study selection criteria, as well as ethnic, regional and other factors could explain the difference in results.

In order to search for COPD-related protein in phases, we compared the proteomic spectra of stable COPD patients, AECOPD patients and healthy controls. The study found that two protein peaks $(3167$ and $4645 \mathrm{~m} / \mathrm{z}$ ) could distinguish stable COPD patients and healthy controls. Sensitivity and specificity were $96.67 \%$ and $96.67 \%$. Three protein peaks (3167, 2963 and $2973 \mathrm{~m} / \mathrm{z}$ ) could distinguish between AECOPD patients and healthy controls, with a sensitivity and specificity of $100 \%$ and $100 \%$. Three protein peaks (5476, 14039 and $2831 \mathrm{~m} / \mathrm{z}$ ) could distinguish between stable COPD patients and AECOPD patients, with a sensitivity and specificity of $93.33 \%$ and $100 \%$, respectively. This shows that the diagnostic models established using distinctive proteomic peaks can discriminate between the different stages of COPD. Therefore, SELDI-TOF-MS technology will not only help the clinical diagnosis of COPD, but also the diagnosis of disease stages. By analyzing the models I, II, and III, we find that one protein peak $(3167 \mathrm{~m} / \mathrm{z})$ of the three models was chosen as the root node. It may be a marker protein of COPD, and its identification and validation may be very important in COPD diagnosis and prevention. In addition, serum protein spectra was compared between stable COPD, AECOPD patients and healthy controls. Five protein peaks (5802, 5839, 9371, 9731 and $11445 \mathrm{~m} / \mathrm{z}$ ) were found to be orderly increasing from healthy controls, stable COPD to AECOPD, and one protein peak $(2303 \mathrm{~m} / \mathrm{z})$ was found to be orderly reducing from healthy controls, stable COPD to AECOPD. These obvious differentially expressed proteins may have a close relationship with the genesis and development of COPD and worthy of further study.

\section{Conclusions}

SELDI-TOF-MS protein chip technology is a quick, easy and practical, high throughput analytic method. It can detect biological markers in COPD, and it shows that the diagnostic models established by distinctive proteomic peaks can discriminate COPD patients from healthy control and can identify different stages of COPD. It will provide a highly accurate approach for diagnosis and clinical staging of COPD. However, the present study is a preliminary research, further studies, increasing the sample size and including other airway obstructive diseases such as asthma into control group, are needed to confirm these results and especially to identify and subsequently validate the discovered protein peaks. In addition, samples from airway locality such as sputum can provide information about both inflammatory cells and mediators present in the airways [29,30]. Sputum reflects the microenvironment of the lung whereas serum reflects a processed proteome; combining data on these samples may provide the necessary means to understand, diagnose and treat COPD [31]. Therefore, in the further study, it is meaningful to include sputum proteomics.

We specially acknowledge Wang XiaoTao and Dong ShuWen of Respiratory Department for their technical support.

1 Mannino D M. COPD: Epidemiology, prevalence, morbidity, mortality, and diseases heterogeneity. Chest, 2002, 12: 121-126

2 Rabe K F, Hurd S, Anzueto A, et al. Global strategy for the diagnosis, management, and prevention of chronic obstructive pulmonary disease: GOLD executive summary. Am J Respir Crit Care Med, 2007, 176: 532-555

3 Halbert R J, Isonaka S, George D, et al. Interpreting COPD prevalence estimates. What is the true burden of disease? Chest, 2003, 123: 1684-1692

4 Celli B R. Update on the management of COPD. Chest, 2008, 133: 1451-1462

5 Elkington $\mathrm{H}$, White P, Higgs R, et al. GP's views of discussion of prognosis in severe COPD. Fam Pract, 2001, 18: 440-444

6 Barnes P, Kleinert S. COPD—A neglected disease. Lancet, 2004, 364 : 564-565.

7 Ohar J A, Sadeghnejad A, Meyers D A, et al. Do symptoms predict COPD in smokers? Chest, 2010, 137: 1345-1353

8 Chenna P R, Mannino D M. Outcomes of severe COPD exacerbations requiring hospitalization. Semin Respir Crit Care Med, 2010, 31: 286-294

9 Woolley J F, Al-Rubeai M. The application of SELDI-TOF mass spectrometry to mammalian cell culture. Biotechnol Adv, 2009, 27: $177-184$

10 Solassol J, Harmand P O, Maudelonde T, et al. Autoantibodies against tumor-related antigens: New tools for early detection of lung cancer. Bull Cancer, 2011, 98: 1419-1430

11 Anderson N L, Anderson N G, Pearson T W, et al. A human proteome detection and quantitation project. Mol Cell Proteomics, 2009, 8: 883-886

12 Broodman I, de Costa D, Stingl C, et al. Mass spectrometry analyses of $\kappa$ and $\lambda$ fractions result in increased number of complementarity-determining region identifications. Proteomics, 2012, 12: 183-191

13 Wang N, Wen X Y, Wang J, et al. Breast cancer-specific serum peptide profiles. Chin Sci Bull, 2009, 54: 421-429

14 Engwegen J Y, Gast M C, Schellens J H, et al. Clinical proteomics searching for better tumour markers with SELDI-TOF mass spectrometry. Trends Pharmacol Sci, 2006, 5: 251-259

15 Issaq H J, Veenstra T D, Conrads T P, et al. The SELDI-TOF-MS approach to proteomics: Protein profiling and biomarker identification. Biochem Biophys Res Commun, 2002, 292: 587-592

16 Espina V, Dettloff K A, Cowherd S, et al. Use of proteomic analysis to monitor responses to biological therapies. Expert Opin Biol Ther, 2004, 4: 83-93 
17 Zou J, Hong G, Guo X, et al. Reproducible cancer biomarker discovery in SELDI-TOF MS using different pre-processing algorithms. PLoS One, 2011, 6: 10

18 Ward D G, Cheng Y, N'Kontchou K G, et al. Changes in the serum proteome associated with the development of hepatocellular carcinoma in hepatitis C-related cirrhosis. Br J Cancer, 2006, 94: 287-292

19 Oh J H, Nandi A, Gurnani P, et al. Proteomic biomarker identification for diagnosis of early relapse in ovarian cancer. J Bioinform Comput Biol, 2006, 4: 1159-1179

20 Lee I N, Chen $\mathrm{C} \mathrm{H}$, Sheu J C, et al. Identification of complement C3a as a candidate biomarker in human chronic hepatitis $\mathrm{C}$ and HCV-related hepatocellular carcinoma using a proteomics approach. Proteomics, 2006, 6: 2865-2873

21 Opstal-van Winden A W, Krop E J, Kåredal M H, et al. Searching for early breast cancer biomarkers by serum protein profiling of prediagnostic serum; a nested case-control study. BMC Cancer, 2011, 11: 381-392

22 Yang Y, Zhao S, Fan Y, et al. Detection and identification of potential biomarkers of non-small cell lung cancer. Technol Cancer Res Treat, 2009, 8: 455-466

23 Bowler R P, Canham M E, Ellison M C. Surface enhanced laser desorption/ionization (SELDI) time-of-flight mass spectrometry to identify patients with chronic obstructive pulmonary disease. COPD, 2006, 3: 41-50
24 Shi L, Zhang J, Wu P, et al. Discovery and identification of potential biomarkers of pediatric Acute Lymphoblastic Leukemia. Proteome Sci, 2009, 7: 7-19

25 Zhang J, Wu X, Shi L, et al. Diagnostic serum proteomic analysis in patients with active tuberculosis. Clin Chim Acta, 2012, 413: 883887

26 Hartwig S, Czibere A, Kotzka J, et al. Combinatorial hexapeptide ligand libraries (ProteoMiner): An innovative fractionation tool for differential quantitative clinical proteomics. Arch Physiol Biochem, 2009, 115: 155-160

27 Sihlbom C, Kanmert I, Bahr H, et al. Evaluation of the combination of bead technology with SELDI-TOF-MS and 2-D DIGE for detection of plasma proteins. J Proteome Res, 2008, 7: 4191-4198

28 Monari E, Casali C, Cuoghi A, et al. Enriched sera protein profiling for detection of non-small cell lung cancer biomarkers. Proteome Sci, 2011, 9: 55-65

29 Fu Y R, Yi Z J, Guan S Z, et al. Proteomic analysis of sputum in patients with active pulmonary Tuberculosis. Clin Microbiol Infect, 2012, doi: 10.1111/j.1469-0691.2012.03824.x

30 Gray R D, MacGregor G, Noble D, et al. Sputum proteomics in inflammatory and suppurative respiratory diseases. Am J Respir Crit Care Med, 2008, 178: 444-452

31 Boggs S E. Protein profiling in respiratory disease: Techniques and impact. Expert Rev Proteomics, 2004, 1: 29-36

Open Access This article is distributed under the terms of the Creative Commons Attribution License which permits any use, distribution, and reproduction in any medium, provided the original author(s) and source are credited. 